\title{
Combining the Prostate Cancer Risk Index (PRIX) with the Presence of Secondary Circulating Prostate Cells to Predict the Risk of Biochemical Failure after Radical Prostatectomy for Prostate Cancer
}

\author{
Nigel P Murray ${ }^{1 *}$, Socrates Aedo ${ }^{1}$, Cynthia Fuentealba ${ }^{2}$, Eduardo Reyes ${ }^{3}$, \\ Omar Jacob ${ }^{2}$
}

\begin{abstract}
Introduction: The use of pre- and post-surgery variables has been used to create nomograms in order to identify patients at high risk of treatment failure. The PRIX nomogram is one such device; we compare the PRIX nomogram with the presence of secondary circulating prostate cells to predict those men who will undergo treatment failure. Methods and Patients: Men who underwent radical prostatectomy for prostate cancer entered the study. The PRIX score was calculated from the total serum PSA pre-surgery, the biopsy Gleason score and clinical stage. Circulating prostate cells were detected from venous blood one month after surgery, using differential gel centrifugation and standard immunocytochemistry with anti-PSA. A test was considered positive when $1 \mathrm{CPC} /$ blood sample was detected. Patients were followed up for five years and biochemical failure was defined as a serum PSA $>0.2 \mathrm{ng} / \mathrm{ml}$. Kaplan-Meier and Cox proportional models were used to calculate survival curves. Results: 321 men participated, of whom 131 (40.8\%) underwent biochemical failure within 5 years. A higher PRIX score was associated with increased failure risk, as was the presence of CPCs. The predictive power of CPCs was significantly higher than the PRIX score. Combining the two methods, for equal PRIX scores, scores but CPC positive had a worse biochemical failure free survival than men with high PRIX scores but CPC negative. For men with PRIX scores of $\geq 4$ the use of CPC detection did not aid in the clinical decision making process. For those with PRIX scores of 0 and 1, CPC detection identified men with a high risk of treatment failure. Conclusions: The combined PRIX/CPC score improved the predictive values of men at high risk of biochemical failure. Both are simple systems that could be incorporated in a general hospital. Further multicenter studies are warranted to confirm these results.
\end{abstract}

Keywords: Prostate cance- PRIX score- circulating prostate cells- biochemical failure

Asian Pac J Cancer Prev, 19 (12), 3375-3381

\section{Introduction}

Radical prostatectomy (RP) is one of the standard therapies for the treatment of localized prostate cancer. Although considered to be localized at the time of treatment, $15-30 \%$ of patients will suffer biochemical relapse within 5 years of surgery (Pound et al., 1999; Han et al., 2003). The risk of treatment failure varies with pre-treatment prostate specific antigen (PSA) levels; the Gleason score and clinical T stage, and with the post-treatment variables of the Gleason score in the surgical specimen and pathological $\mathrm{T}$ stage. These variables have been combined to produce statistical models to predict the risk of biochemical relapse, such as the D'Amico risk classification (D'Amico et al., 1998), the Stephenson nomogram (Stephenson et al., 2006) and the
Cancer of the Prostate Risk Assessment (CAPRA) score (Cooperberg et al., 2005). The identification of patients at high risk of treatment failure is important as early use of androgen blockade or radiotherapy has a greater benefit than when used later in the disease (Messing et al., 2006; Thompson et al., 2009) and to identify patients with a lower risk, who could be spared the potential side effects of treatment.

The Prostate Cancer Risk Index (PRIX) was developed as a simple scoring system using three pre-treatment variables, PSA at diagnosis, Gleason score of the prostate biopsy and clinical T stage. Each variable is assigned a score and the sum of these scores $0-6$ is used to stratify the patients according to risk of subsequent biochemical failure (Yoshioka and Inoue, 2007). The PRIX score has been externally validated (Yoshida et al., 2011). 
Recent studies have shown that men positive for secondary circulating prostate cells (CPCs), that is those detected after RP, have a seven fold increase of biochemical failure (Murray et al., 2013) and thus identify a high risk group before increases in serum PSA are detected. We present a prospective study of Chilean men who have undergone radical prostatectomy and compare the predictions of the PRIX score with the presence of secondary CPCs and clinical outcomes.

\section{Materials and Methods}

\section{Methods and Patients}

Between 2008 and 2014, men who underwent open retro-pubic radical prostatectomy for prostate cancer were enrolled in the study. Men who had received hormonal treatment or were considered for adjuvant radiotherapy or hormonal treatment post radical prostatectomy were excluded from the study. For each patient, after giving informed written consent, the following were recorded: date of surgical treatment, age at surgery, total serum PSA at diagnosis (measured using the Siemens Advia CentaurXR ${ }^{\circledR}$ assay), Clinical Stage (was determined according to the 2002 TMN classification) and Gleason Scores (from RP specimen analyzed by a dedicated genitourinary pathologist according to the Gleason system).

Total serum PSA levels and the presence/absence of CPCs were measured one month after RP. During followup total serum PSA levels were measured three monthly for the first two years and then six monthly. Biochemical relapse was defined as a PSA level of $>0.2 \mathrm{ng} / \mathrm{ml}$ on two separate occasions at least four weeks apart.

The PRIX score with a range of $0-6$ points was determined as follows; a PSA level at diagnosis of $<10.0 \mathrm{ng} / \mathrm{ml}$ was assigned 0 points, $10.0-20.0 \mathrm{ng} / \mathrm{ml}$ was assigned 1 point and $>20.0 \mathrm{ng} / \mathrm{ml} 2$ points. Gleason scores at biopsy of $\leq 6,7$ and $\geq 8$ were assigned 0,1 and 2 points respectively. Clinical stages T-T2a, T2b-T2c and T3-T4 were assigned 0,1 and 2 points respectively.

\section{Detection of secondary CPCs}

One month after surgery, using EDTA as an anticoagulant (BD-Vacutainer $\left.{ }^{\circledR}\right)$ an $8 \mathrm{~mL}$ of venous blood was taken, maintained at $4^{\circ} \mathrm{C}$ and processed within 48 hours.

Differential gel centrifugation using Histopaque 1,077 (Sigma-Aldrich) was used to obtain mono-nuclear cells, which were washed, and re-suspended in a $100 \mu \mathrm{L}$ aliquot of autologous plasma. $25 \mu \mathrm{L}$ aliquots were used to make 4 slides (silanized, DAKO, USA). These were air dried for 24 hours and then fixed (70\% ethanol, 5\% formaldehyde, and $25 \%$ phosphate buffered saline $\mathrm{pH} 7.4$ ) for five minutes and finally washed three times in PBS pH 7.4.

\section{Immunocytochemistry}

Anti-PSA monoclonal antibody, clone 28A4 (Novocastro Laboratory, UK) was used to detect CPCs, and an alkaline phosphatase-anti alkaline phosphatase based system (LSAB2, DAKO, USA), with new fuchsin as the chromogen to identify them. Samples positive for
CPCs were incubated with anti-CD45 clone (DAKO, USA) and processed with a peroxidase based system (LSAB2, DAKO, USA) with DAB (3,3 diaminobenzidine tetrahydrochloride) as the chromogen.

The ISHAGE (International Society of Hemotherapy and Genetic Engineering) were used to define a CPC (Borgen et al., 1999), as a cell that expressed PSA but not CD45. Slides were analyzed manually to determine if CPCs were present or absent. A test was considered positive when at least one cell $/ 8 \mathrm{~mL}$ of blood was detected. In order to assess the reliability/reproducibility of CPC detection using this method, thirty samples in duplicate were analyzed by three different trained cytologists in a blinded fashion to determine the presence or absence of CPCs. A test was defined as positive or negative, based on the theoretical reason that if CPCs were detected independent of the number, the implication was that minimal residual disease was present. (Figure 1a and Figure 1b)

\section{Statistical analysis}

The analysis was performed using the program Stata (Stata/SE 14.0 for Windows, Stata Corp Lp, 20159, describing according to the nature and distribution of the quantitative and ordinate variables with measurements of central tendency (mean and median) and of dispersion using the inter-quartile range (IQR) and standard deviation (SD) (Rosner, 2015). The Shapiro-Wilk Test was used to define the null hypothesis with respect to the normal distribution. The nominal dichotomous variables were described as proportions with their respective confidence intervals (Rosner, 2015).

In the thirty subjects assessed by three different cytologists the observed and expected intra and inter-observer agreements were determined, as well as the Kappa statistic to assess the intra and inter-observer reliability of the test for the presence or absence of CPCs.

Age, PSA at diagnosis, Gleason Score, Clinical Stage, PRIX Score and presence of secondary CPC were compared, to presence of relapse (Rosner, 2015).

For all the men studied the survival of biochemical failure at 3 and 5 years was described using the Survival Curves of Kaplan-Meier (Cleves et al., 2010).

Evaluating the validity of the PRIX score for our study group, we analyzed the calibration of the data using partial validation (the closeness of fit of the prediction of possible survival) (Royston, 2015); where the prognostic index (Neperiano logarithm of the hazard ratio) obtained from published data (Yoshida et al., 2011) is applied to the PRIX score of the observed study data. The aforesaid published survival is compared with our observed survival using the same categorization of patients. The discrimination of a prognostic model reflects its ability to distinguish between patient outcomes. For PRIX score for our study group; the discriminatory power is evaluated using Harrell's C concordance test (Harrell et al., 1982).

From the predictors: Age, Prix Score (The scores are embedded as dummy variables including a value from zero to five) and the presence of secondary CPC a multivariable cox regression was performed in order to evaluate the relapse predicted during the five-year follow-up (Cleves 
et al., 2010). The cox regression was conducted by means of a stepwise backward selection approach. The constructed final model was established with predictors whose coefficients show statistical significance ( $\mathrm{p}$ value $<0.05$ ) and the fulfillment of assumptions (this includes proportional risk, adequate calibration and discrimination) (Cleves et al., 2010; Rosner, 2015; Royston 2015).

In this way the calibration aspect of the model refers to agreements between the predicted outcome and observed outcome (Royston, 2015). For final model, this was assessed using graphical methods including the observed versus predicted values for the probabilities, predictions and cumulative hazard of Cox-Snell residuals (Cleves et al., 2010). Also, we performed the comparison of survival for Cox and Kaplan-Meier models (Cleves et al., 2010).

The discrimination of a prognostic model reflects its ability to distinguish between patient outcomes. For the final model we calculated, the Harrell's C discrimination index (Harrell, 2001); the which is scored on a scale of 0 to 1 . This can be taken to mean that if two cases are drawn at random, the $\mathrm{c}$ statistic is the probability that the person who survives the longest had the highest predicted survival.

Values near 0 or 1 indicate perfect discrimination. Values of $0.50-0.60$ indicate a very poor predictive model; $0.60-0.75$ is defined as acceptable; $0.75-0.90$ is defined as good; $0.90-0.97$ as very good and $>0.97$ as excellent.

To evaluate the clinical utility of final model in the prediction of relapse, a decision curve analysis was used, to evaluate the clinical consequences of the predictive combined model (Vickers et al., 2008).

\section{Ethical considerations}

The study was approved by the local ethics committee and fully complied with the Declaration of Helsinki.

\section{Results}

\section{Descriptive statistical groups}

Three hundred and twenty one men participated in the study with a mean age of $65.5 \pm 8.3$ years. The PSA at diagnosis showed an asymmetric distribution with a median and IQR respectively of $5.48 \mathrm{ng} / \mathrm{ml}$ and $3.26 \mathrm{ng} /$ ml. 272/321 (85\%) of patients had a total serum PSA of $<10 \mathrm{ng} / \mathrm{ml}$ at diagnosis, 39/321 (12\%) a PSA between 10-20ng/ml and 10/321 (3\%) a PSA $>20 \mathrm{ng} / \mathrm{ml}$. 223/321 $(70 \%)$ men had a Gleason score of $\leq 6,60 / 321,(19 \%)$ a Gleason score of 7 and 38/321 (1159 a Gleason score of $\geq 8$. In terms of clinical staging 109/321 (34\%) had stage T1c-T2a, 147/321 (46\%) had clinical stage T2b-T2c and $65 / 321(20 \%)$ had clinical stage T3a. A total of 131/321 (40.4\%) men underwent biochemical relapse during the study period.

The intra and inter-observer reliability of the test for the presence or absence of CPCs.

The presence or absence of CPC in thirty subjects was analyzed in duplicate by three different cytologists, the expected and observed inter operator agreement were respectively $51.6 \%$ and $88.9 \%$. The expected and observed intra operator agreement was respectively $52.2 \%$ and $90.0 \%$. The Kappa statistic for inter and intra
Table 1. Characteristics of 321 Men with and without Biochemical Failure Treated by Radical Prostatectomy for Prostate Cancer

\begin{tabular}{|c|c|c|c|}
\hline Variable & $\begin{array}{l}\text { Men without } \\
\text { BF } n=190\end{array}$ & $\begin{array}{l}\text { Men with } \\
\text { BF } n=131\end{array}$ & p-value \\
\hline $\begin{array}{l}\text { Age* (years) } \\
\text { Mean } \pm \text { SD }\end{array}$ & $64.71 \pm 8.07$ & $\begin{array}{c}66.52 \pm \\
8.64\end{array}$ & $0.028^{\mathrm{a}}$ \\
\hline $\begin{array}{l}\mathrm{PSA}^{*}, \mathrm{ng} / \mathrm{ml} \\
\text { Median; IQR }\end{array}$ & $5.21 ; 1.89$ & $6.03 ; 3.84$ & $<0.001^{\mathrm{b}}$ \\
\hline Gleason Score* & $5 ; 1$ & $7 ; 1$ & $<0.001^{\mathrm{b}}$ \\
\hline \multicolumn{4}{|l|}{ Clinical stage } \\
\hline T1c-T2a n (\%) & $96(50.53)$ & $13(9.92)$ & \\
\hline T2b-T2c n (\%) & $76(40.00)$ & $71(54.20)$ & $<0.001^{\mathrm{c}}$ \\
\hline T3a n (\%) & $18(9.47)$ & $47(35.88)$ & \\
\hline $\begin{array}{l}\text { PRIX Score } \\
\text { *Median; IQR }\end{array}$ & $1 ; 1$ & $2 ; 2$ & $<0.001^{\mathrm{b}}$ \\
\hline $\begin{array}{l}\text { CPC positive } \\
\text { n }(\%)\end{array}$ & $18(9.47)$ & $109(83.21)$ & $<0.001^{\mathrm{c}}$ \\
\hline
\end{tabular}

*Shapiro-Wilk Test $\mathrm{p}$ valor $<0.15$; PSA, serum total PSA at diagnosis; $\mathrm{SD}$, standard deviation; IQR, interquartile range; CPC, Presence of secondary circulating prostate cell; a, Student's t-test one tailed assuming equal variance (Variance ratio test p-value >0.05); b, MannWhitney Test one tailed; c, Pearson's Chi squared test a two tailed.

operator reliability for the presence or absent CPC were respectively 0.77 (CI 95\%: 0.58 to 0.82 ) and 0.79 (CI 95\%:0.66 to 0.92 ).

\section{Biochemical Failure}

There was a significant statistical (p-value $<0.05$ ) association between biochemical failure and age, PSA at diagnosis, Gleason Score, Clinical Stage, Prix Score and

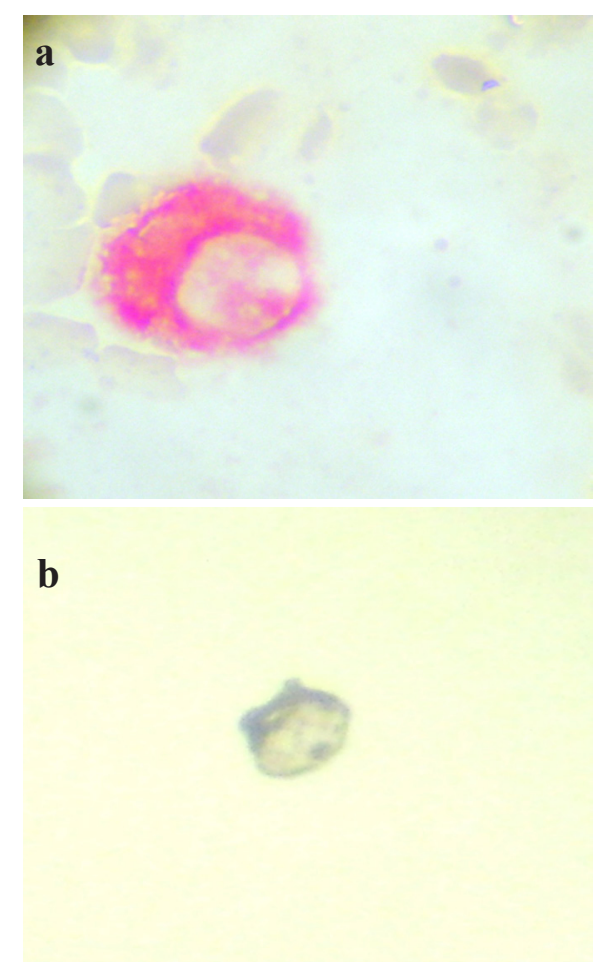

Figure 1. Photo Circulating Prostate Cell 
Table 2. Biochemical Failure Free Progression at 3 and 5 years. Comparing predicted (according to the models of Coxa) versus observed survival (model Kaplan-Meier), in 321 men treated by radical prostatectomy.

\begin{tabular}{|c|c|c|c|c|}
\hline & \multicolumn{2}{|c|}{$\begin{array}{c}\text { Survival to } 3 \text { years } \\
\text { Percentage (CI:95\%) }\end{array}$} & \multicolumn{2}{|c|}{$\begin{array}{c}\text { Survival to } 5 \text { years } \\
\text { Percentage (CI:95\%) }\end{array}$} \\
\hline & Observed & Predicted a & Observed & Predicted a \\
\hline \multirow[t]{2}{*}{ CPC (-) PRIX $\geq 2$} & $96.55 \%$ & 95.68 & 92.84 & 92.65 \\
\hline & (77.95 to 99.51$)$ & (89.41 to 98.27 ) & (74.25 to 98.16$)$ & (82.60 to 97.00 ) \\
\hline \multirow[t]{2}{*}{ CPC (-) PRIX 1} & $100^{\mathrm{b}}$ & 98.43 & 94.29 & 97.3 \\
\hline & & (95.82 to 99.41$)$ & (83.32 to 98.12 ) & (93.04 to 98.96 ) \\
\hline \multirow[t]{2}{*}{ CPC (-) PRIX 0} & $100^{\mathrm{b}}$ & 98.94 & $100^{\mathrm{b}}$ & 98.18 \\
\hline & & (96.93 to 99.64 ) & & (94.85 to 99.36 ) \\
\hline \multirow[t]{2}{*}{ CPC (+) PRIX $\geq 2$} & 39.53 & 39.87 & 21.68 & 20.44 \\
\hline & (28.95 to 49.92$)$ & (29.84 to 49.70$)$ & (13.42 to 31.24$)$ & (12.79 to 29.35 ) \\
\hline \multirow[t]{2}{*}{ CPC (+) PRIX 1} & 66.67 & 71.87 & 62.96 & 56.53 \\
\hline & (45.71 to 81.06 ) & (55.70 to 82.99 ) & (42.12 to 78.07 ) & (37.34 to 71.87 ) \\
\hline \multirow[t]{2}{*}{ CPC (+) PRIX 0} & 85.71 & 80.15 & 52.91 & 68.24 \\
\hline & (53.94 to 96.22$)$ & (60.14 to 90.82 ) & (22.63 to 76.13 ) & (42.19 to 84.44 ) \\
\hline
\end{tabular}

$\overline{\mathrm{CPC}}(+)$, presence of secondary circulating prostate; CPC (-) rated, absence of secondary circulating prostate.

presence of secondary CPC (Table 1).

After 3 and 5 years of follow up, the Kaplan-Meier biochemical failure free survival for the whole group was respectively $79.10 \%$ (95\% CI: 74.00 to 83.32 ), and $70.33 \%$ (95\% CI: 64.61 to 75.30$)$.
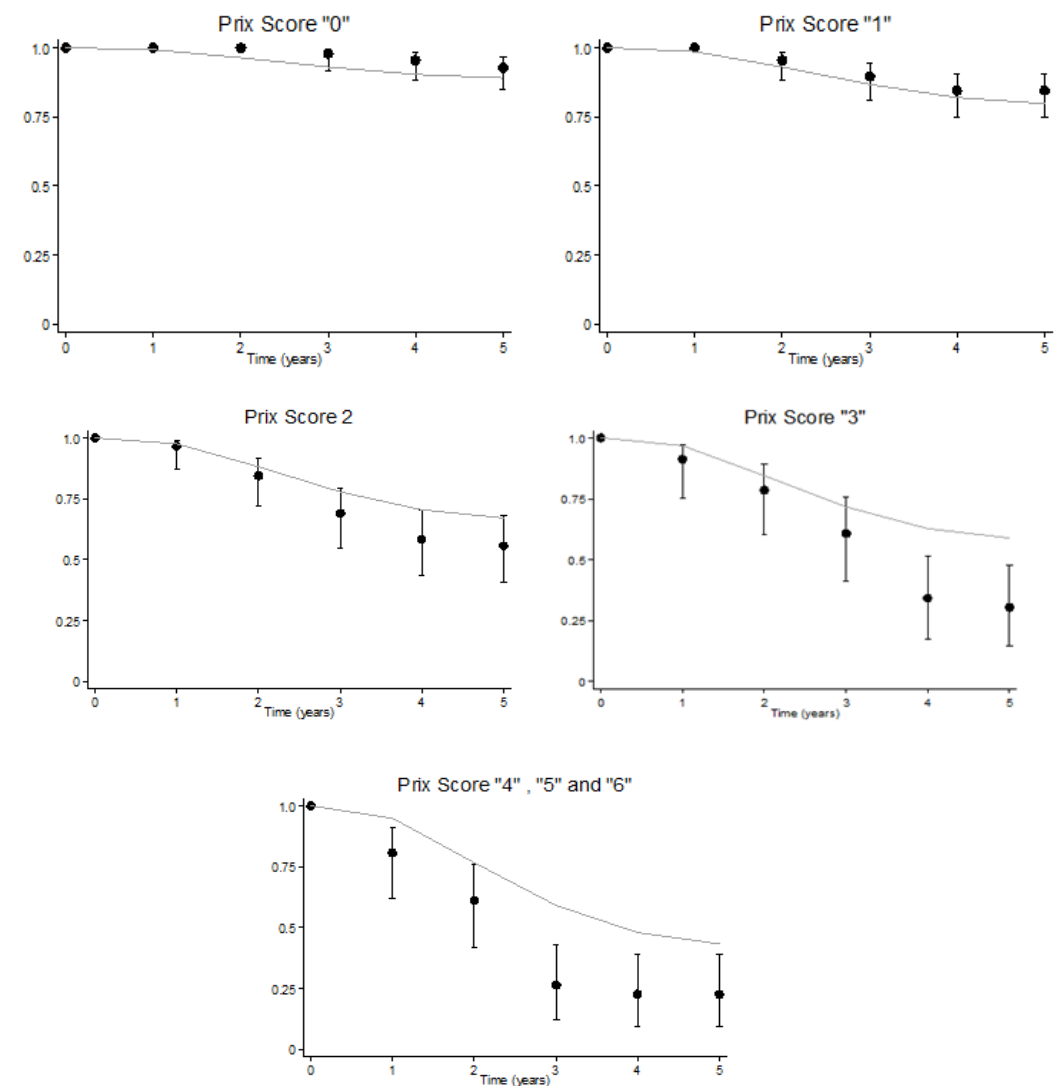

Figure 2. Partial Calibration of a Cox Model with the Prognostic Index Estimated from the Published Regression Coefficient and Evaluated on the Validation Dataset with Re-Estimation of the Baseline Cumulative-Hazard Function. Smooth dashed lines represent predicted survival probabilities, and vertical capped lines represent Kaplan-Meier estimates with $95 \%$ confidence intervals. Five prognosis groups were plotted 


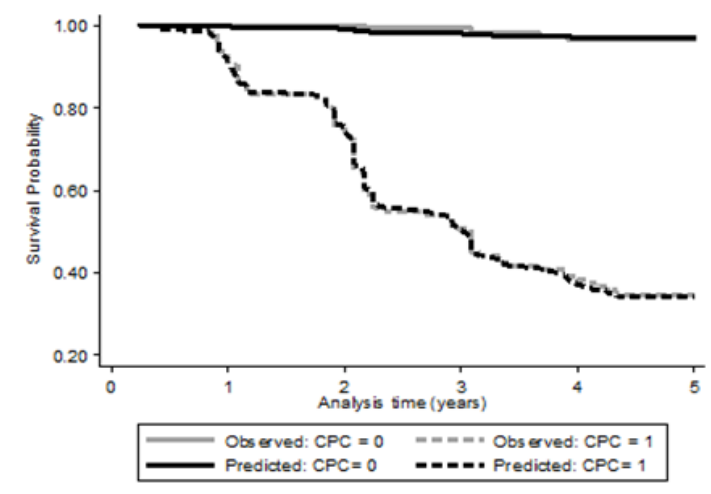

a) Survival Curves at 5 years CPC (-) versus CPC (+) Men

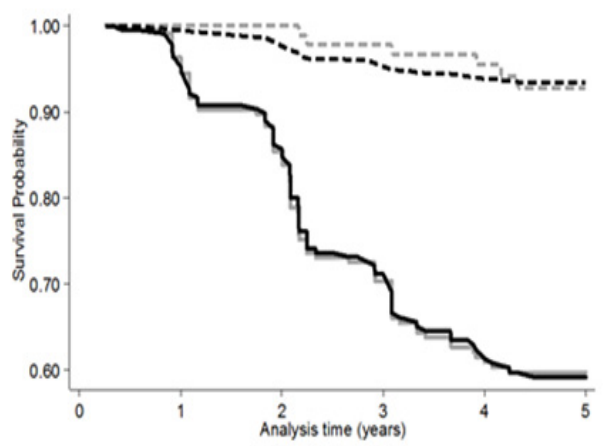

b) Survival Curves at 5 Years in Men with PRIX $=0$, CPC (-) (dotted line)

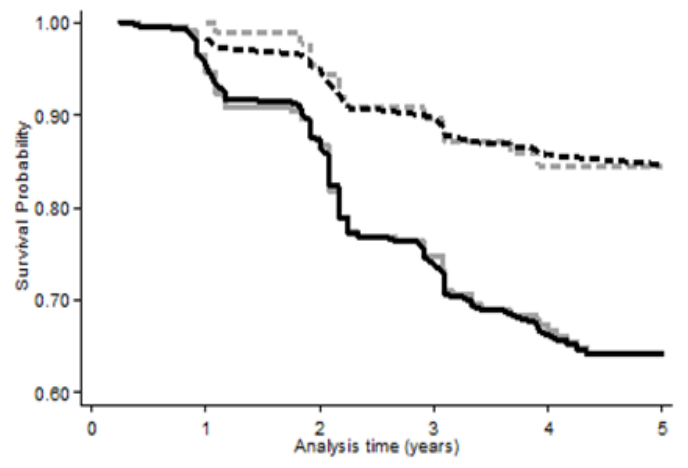

c). Survival Curves at 5 Years in Men with PRIX=1, CPC (-) (dotted line) versus CPC (+) unbroken line

Figure 3. Biochemical Failure Survival for Prostate Cancer at 5 Years According to Presence of Secondary CPCs, PRIX Score of 0 and 1 Comparing the Observed Survival (Kaplan-Meier) with Predicted Survival (Cox Model) in 321 Subjects Treated by Radical Prostatectomy.

(good), between the predicted survival and the observed survival.

Kaplan-Meier survival curves showed that men CPC negative had a better prognosis than men $\mathrm{CPC}$ positive for the same PRIX score, Figure 2 shows the survival curves of CPC negative versus CPC positive for the whole group and in those men PRIX 0 and PRIX 1.

\section{Assessment of the Calibration and discrimination for selected final model}

The cumulative hazard of Cox-Snell residuals for the model studied, showed adequate a goodness of fit.

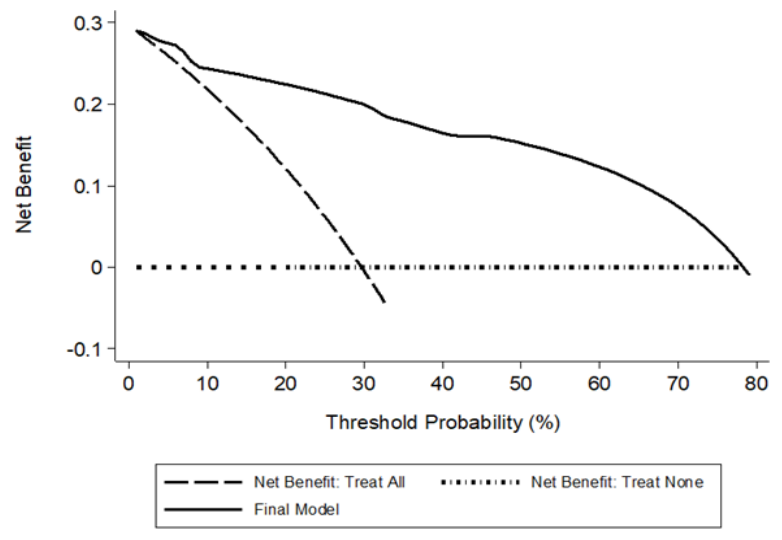

Figure 4. Decision Curve Analysis Comparing Models Using CPC, PRIX Score Values Zero and One, in 321 men treated by radical prostatectomy for prostate cancer. *The Final Model is based on Cox's proportional hazards risk, using dummy variables: a) presence of secondary circulating prostate, b) presence of PRIX Score equal zero and c) presence of PRIX Score equal one.

During five years, the comparing predicted (according to the model of Cox) versus observed survival (model Kaplan-Meier) showed agreement (Table 2, Figure 3). The Harrell's C concordance coefficient was 0.86 .

\section{Assessment clinical utility for selected final model}

The decision curve analysis, showed that for probability threshold values between 0 and $80 \%$ the final model built with cox regression, is most useful than strategies of treat all or none (Figure 4).

The decision curve analysis, showed that for probability threshold values between 0 and $80 \%$ the final model built with cox regression, is most useful than strategies of treat all or none (Figure 4).

\section{Discussion}

From the clinical point of view, after surgery for prostate cancer the question arises of which patients should be considered for adjuvant therapy owing to a high risk of treatment failure? The PRIX score incorporates known risk factors for treatment failure, however the authors did not include recommendations as to treatment options associated with differing PRIX scores (Yoshida et al., 2011). This differs from the CAPRA score (Cooperberg et al., 2011) where patients classified as low risk (a score of $\leq 2$ ) the treatment recommended is observation and for those considered to be at intermediate and high risk (a score of $\geq 3$ ) may be offered treatment to decrease the risk of biochemical recurrence. As with the CAPRA score the PRIX uses clinical variables which are fixed and thus if the patient receives additional therapy the score is no longer valid. Neither does the PRIX score predict whether the failure will be local or systemic in origin and therefore does not help in the clinical decision of which adjuvant therapy should be considered; radiotherapy to the prostate bed or systemic androgen blockade.

In this article, we used the term circulating prostate cells; in general, the term circulating tumor cells has been used to describe Epithelial Cell Adhesion Molecule 
(EpCAM) expressing cells found in the blood of cancer patients. The cells identified in this article express PSA, highly specific for prostate cells and as such, we used the more specific definition of circulating prostate cells. Due to the epithelial mesenchymal transition seen in cancer cells not all circulating tumor cells express EpCAM or cytokeratins (Raimondi et al., 2011). Circulating cells that express cytokeratins or EpCAM (epithelial cell adhesion molecule) are not prostate specific, may be detected in benign diseases such as inflammatory bowel disease (Pantel et al., 2012). Anti-PSA was chosen to detect CPCs, based on its specificity for prostate tissue. The expression of PSA is dependent on a functioning androgen receptor and as such the presence of androgen blockade theoretically could modify the sensitivity of this assay. In this study none of the men were receiving androgen blockade as part of their treatment.

We defined prostate cells detected in blood after curative treatment as secondary circulating prostate cells. Those present before surgery have been defined as primary circulating prostate cells. For a cancer to be able to produce metastasis, the cancer cells have to disseminate from the primary tumour, survive in the circulation and to able to adhere to the vascular endothelium at a distant site before invading the distant tissue. If not all CPCs are able to adhere and invade distant sites, then complete tumour removal at the time of surgery would imply curative therapy and in the clinical would be seen as better survival rates. Recent studies have shown that the mere presence of primary CPCs is not a good prognostic factor for biochemical failure free survival (Meyer et al., 2016; Murray et al., 2016; Murray et al., 2016a). The detection of circulating tumour cells using combined nested reverse transcriptase polymerase chain reaction pre-radical prostatectomy also failed to predict biochemical failure (Thomas et al., 2002).

Secondary CPC detection also gives important clinical information on the risk of treatment failure, but equally does not predict whether initial treatment failure will be local or systemic. Differing from the PRIX score it is a dynamic parameter and can be repeated during followup and can be used to monitor treatment. Furthermore, phenotypic analysis of CPCs may help to determine which type of systemic therapy may have a greater possibility of success (Murray et al., 2011).

We designed the test to be positive or negative; firstly on the theoretical basis that if CPCs were present the inference was that there was minimal residual disease, independent of whether this disease was local or distant from the prostate bed. Secondly a positive/negative results aids in the clinical decision and finally the intra and inter-observer variability was low. The kappa indices and agreements intra and inter operators showed that our technique for the absence or presence of CPC have adequate reliability when used by different trained operators.

Prediction models are based on the statistical analysis of outcomes in a cohort of patients and then validated. The original authors of the PRIX prediction model developed this model based on the correspondence to other prediction models (8) and it has been externally validated in a group of Japanese patients (9). In our group of Chilean men, the PRIX score of 4 or more showed an poor adjust (bad calibration) between the predicted and observed survival, which could be due to the original population $(n=519$ subjects) used as a standard for the partial validation showed a small number of subjects 13, 1 and 1 for respectively score PRIX 4, 5 and 6 (19); conversely in our study 19, 9 and 4 subjects were respectively PRIX score 4, 5 and 6 . The observed survival was significantly worse than the predicted survival. Patients in this group, who were nearly all CPC positive, would be classified as high risk according to the European Association of Urology Prostate Cancer Guidelines (EAU, 2016). These guidelines would recommend adjuvant therapy and from this study it would suggest that men with a PRIX score of 4-6 should be considered for adjuvant therapy. In this group the addition of CPC testing would not add to the decision making process.

The combined model using both PRIX and secondary CPC changes risk groups in a significant number of patients. For the same PRIX score of 0,1 and $\geq 2$ the prognosis of CPC positive patients was significantly worse than CPC negative patients. There was a subgroup of low risk PRIX $=0$ patients who were CPC $(+)$, who had a significantly worse prognosis. While a group of patients with a high risk PRIX score defined as a score $\geq 2$ who were CPC (-) and had a much better prognosis. These patients had a higher 5 year PFS than men with a PRIX $=0 \mathrm{CPC}(+)$. Due to this, we believe that the addition of CPC detection gives clinically significant information of who may be eligible for adjuvant therapy, and inversely who may not need adjuvant therapy. Differing from the group PRIX 4-6 the addition of CPC testing adds clinically significant information that could be used to define patient management. In low risk groups that would not be considered for adjuvant therapy, the use of CPC detection appears to identify men with a significantly increased risk of treatment failure.

A decision analysis curve was used to determine the clinical consequences of incorporating the combined model in predicting the risk of biochemical failure. Differing from traditional statistical measures of a biomarker or test such as specificity, sensitivity, area under the curve, where it is not clear how high the statistical measure should be to warrant its clinical use, the decision analysis curve attempts to address this problem by incorporating the clinical consequences of using the test or biomarker. The basic interpretation of a decision curve is that the strategy with the highest net benefit at a particular threshold has the highest clinical value. The use of the combined CPC marker had no benefit over the PRIX model up to a threshold of $10 \%$ probability of biochemical failure. Between $10 \%$ and $80 \%$ the combined model was superior, that is to say there was a net benefit using the marker in predicting future biochemical failure.

The study should be confirmed using a larger population and multiple centers, this would define inter-center variability and determine the test's use in the routine immunocytochemical laboratory of a general hospital.

In conclusion, the present study shows that use of 
pretreatment clinical variables in combination with the detection of secondary CPCs detected using standard immunocytochemistry can stratify patients into distinct groups based on the risk of future biochemical failure. These groups may aid in the clinical decision to determine the need for early adjuvant therapy in men with low and intermediate risk prostate cancer. In men with high risk prostate cancer the use of CPC detection does not aid in the clinical decision making process.

\section{Funding}

The study was supported by Hospital de Carabineros de Chile Research Grant

\section{Conflict of Interests}

Dr Murray reports having received consultancy fees from VIATAR CTC solutions, the rest of the authors report no potential conflicts of interests.

\section{Acknowledgements}

The authors wish to thank Mrs. Ana Maria Palazuelos for help in the writing of this manuscript.

\section{References}

Borgen E, Naume B, Nesland JM, et al (1999). Standardization of the immunocytochemical detection of cancer cells in BM and blood: Establishment of objective criteria for the evaluation of immunostained cells. Cytotherapy, 1, 377-88.

Cleves M, Gutierrez R, Gould W, Mrachenko Y (2010). An introduction to survival analysis using stata. Third Edition. Texas: Stata Press; pp 412.

Cooperberg MR, Pasta DJ, Elkin EP, et al (2005). The University of California, San Francisco cancer of the prostate risk assessment score: a straightforward and reliable preoperative predictor of disease recurrence after radical prostatectomy. J Urol, 173, 1938-42.

Cooperberg MR, Hilton JE, Carroll PR (2011). The CAPRA-S score a straightforward tool for improved prediction of outcomes after radical prostatectomy. Cancer, 117, 5039-46.

D`Amico AV, Whittington R, Malkowicz SB, et al (1998). Biochemical outcome after radical prostatectomyt, external beam radiation therapy ot interstitial radiation therapy for clinically localized prostate cancer. JAMA, 280, 969-74.

European Association of Urology Prostate Cancer Guidelines, 2016 www.uroweb.org.

Han M, Partin AW, Zahurak M, et al (2003). Biochemical (prostate specific antigen) recurrence probability following radical prostatectomy for clinically localized prostate cancer. J Urol, 169, 517-23.

Harrell FE Jr, Califf RM, Pryor DB, Lee KL, Rosati RA (1982). Evaluating the yield of medical tests. JAMA, 247, 2543-6.

Harrell FE Jr (2001). Regression modeling strategies with applications to linear models, logistic regression, and survival analysis. New York: Springer- Verlag; pp 568.

Messing EM, Manola J, Yao J, et al (2006). Immediate versus deferred androgen deprivation treatment in patients with node positive prostate cancer after radical prostatectomy and pelvic lymphadenectomy. Lancet Oncol, 7, 472-9.

Meyer CP, Pantel K, Tennstedt P, et al (2016). Limited prognostic value of preoperating circulating tumor cells for early biochemical recurrence in patients with localized prostate cancer. Urol Onc, 34, 11-6.

Murray NP, Badinez L, Dueñas R, Orellana N, Tapia P (2011).
Positive HER-2 protein expression in circulating prostate cells and micro-metastasis, resistant to androgen blockade but not DES. Ind J Urol, 27, 200-7.

Murray NP, Reyes E, Orellana N, et al (2013). Secondary circulating prostate cells predict biochemical failure after radical prostatectomy and without evidence of disease. Sci World J, http://dx.doi.org/10.1155/2013/762064

Murray NP, Aedo S, Fuentealba C, et al (2016). Limited improvement of incorporating primary circulating prostate cells with the CAPRA score to predict biochemical falire free outcome of radical prostatectomy for prostate cancer. Urol Onc, 34, 430.e17-25.

Murray NP, Reyes E, Fuentealba C, Jacob O (2016a). Efficacy of using sequential primary circulating prostate cell detection for initial prostate biopsy in men suspected of prostate cancer. Asian Pac J Cancer Prev, 17, 3385-90.

Pantel K, Denève E, Nocca D, et al (2012). Circulating epithelial cells in patients with benign colon disease. Clin Chem, $\mathbf{5 8}$, 936-40.

Pound CR, Partin AW, Eisenberger MA, et al (1999). Natural history of progression after PSA elevation following radical prostatectomy. JAMA, 281, 1591-7.

Raimondi C, Gradilone A, Naso G, et al (2011). Epithelial-mesenchymal transition and stemness features in circulating tumor cells from breast cancer patients. Breast Cancer Res Treat, 130, 449-55.

Rosner B (2015). Fundamentals of biostatistics. Eighth Edition. Boston: Cengage Learning; pp 927.

Royston P (2015). Tools for checking calibration of a Cox model in external validation: prediction of population-averaged survival curves based on risk group. Stata J, 15, 275-91.

Shariat SF, Karakiewicz PI, Suardi N, Kattan MW (2008). Comparison of nomograms with other methods for predicting outcomes in prostate cancer: a critical analysis of the literature. Clin Cancer Res, 14, 4400-7.

Stephenson AJ, Scardino PT, Eastham JA, et al (2006). Preoperative nomogram predicting the 10 year probability of prostate cancer recurrence after radical prostatectomy. $J$ Natl Cancer Inst, 98, 715-7.

Thomas J, Gupta M, Grasso Y, et al (2002). Preoperative combined nested RT-PCR for PSA and PMSA does not correlate with pathologic stage or biochemical failure in patients with localized prostate cancer undergoing radical prostatectomy. J Clin Oncol, 20, 3213-8.

Thompson IM, Tangen CM, Pardelo J, et al (2009). Adjuvant radiotherapy for pathological T3N0M0 prostate cancer significantly reduces risk of metastasis and improves survival: long term follow up of a randomized clinical trial. J Urol, 181, 956-62.

Vickers AJ, Cronin AM, Elkin EB, Gönen M (2008). Extensions to decision curve analysis, a novel method for evaluating diagnostic tests, prediction models and molecular markers. BMC Med Inform Decis Mak, 8, 53.

Yoshida T, Nakayama M, Matsuzaki K, et al (2011). Validation of the Prostate Cancer Risk Index (PRIX): A simple scoring system to predict risk of biochemical relapse after radical prostatectomy for prostate cancer. Jpn J Clin Oncol, 41, 1271-6.

Yoshioka Y, Inoue T (2007). Prostate Risk Index (PRIX) as a new method of risk classification for clinically localized prostate cancer. Strahlenther Onkol, 183, 490-6.

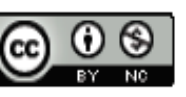

This work is licensed under a Creative Commons AttributionNon Commercial 4.0 International License.

Asian Pacific Journal of Cancer Prevention, Vol 19 